Research Article

\title{
Experiment on Direction of Arrival Estimation of Atmospheric Spark Discharge Plasma Using Combined Time-Domain Antenna
}

\author{
Jiangzehui Yan $\mathbb{D}^{1},{ }^{1}$ Luping Xu $\mathbb{D}^{1},{ }^{1}$ Weihao Tie, ${ }^{2}$ and Dan Jiang ${ }^{3}$ \\ ${ }^{1}$ School of Aerospace Science and Technology, Xidian University, Xi'an, China \\ ${ }^{2}$ China Academy of Space Technology (Xi'an), Xi'an, China \\ ${ }^{3} X i$ 'an Electronic Engineering Research Institute, Xi'an 710100, China \\ Correspondence should be addressed to Luping Xu; lupingxu_xidian@163.com
}

Received 3 June 2020; Revised 15 August 2020; Accepted 24 August 2020; Published 27 September 2020

Academic Editor: Mauro Parise

Copyright (c) 2020 Jiangzehui Yan et al. This is an open access article distributed under the Creative Commons Attribution License, which permits unrestricted use, distribution, and reproduction in any medium, provided the original work is properly cited.

Apart from interfering in the communication system of an aircraft, electromagnetic pulses (EMPs) radiated from spark discharge plasma, which is generated during high-speed flight, can also be utilized in passive detection. In order to validate this idea, an experiment on direction of arrival (DOA) estimation of a spark discharge plasma target using its radiated EMPs is carried out in this paper. A combined time-domain antenna is designed based on the model of spark discharge process and is used to receive the radiated EMPs during the experiment. According to the experimental results, the DOA estimation system with combined antenna is able to obtain the direction information of a spark discharge plasma. Results also show that the estimation performance of elevation angle is better when the actual elevation angle of the discharge plasma target is higher, while the estimation performance of azimuth is opposite. The azimuth angle of a target has very little influence on the DOA estimation. Moreover, the estimation error can be reduced effectively by increasing the aperture size of receiving array. The previously mentioned results provide an approach to locate the discharge plasma source using radiated EMPs with passive detection techniques.

\section{Introduction}

Discharge plasma can be generated on the surface of an aircraft during high-speed flight due to the collision between the air and aircraft itself [1] and brings about unexpected interferences, such as communication blackout and electronic equipment damaging [2-4]. However, the discharge process and discharge plasma can also be utilized: When discharge process occurs on the surface of an aircraft, electromagnetic pulses (EMPs) are generated and radiated to free space. With properly designed receiving element, the direction of arrival (DOA) of the aircraft can be estimated by a passive detection system using the radiated EMPs theoretically. In our previous study, the radiation characteristics of the EMPs from spark discharge plasma in time domain as well as frequency domain has been investigated, and a combined dipole-loop antenna model was proposed as an equivalent model of the entire spark discharge process [5]. So, in this paper, an experiment on spark discharge plasma
DOA estimation is carried out to validate the idea of utilizing the radiated EMPs in passive detection. During the experiment, a combined time-domain antenna, which is designed based on the discharge model, was applied as receiving element.

Experiments of plasma radiation measuring have been performed in many laboratories, and different kinds of measuring equipment are employed, including antennas. Pearlman and Dahlbacka observed ratio frequency (RF) emissions from laser-produced plasma with two dipole antennas [6]. Wang et al. used mini-biconical antenna to investigate the characteristics of RF electromagnetic radiation from laser-induced air plasma [7]. Forestier et al. studied the RF emissions from femtosecond filaments in air with two planar integrated antennas [8]. Aspiotis et al. utilized two ridged waveguide horn antennas to detect $1-18 \mathrm{GHz}$ and $18-40 \mathrm{GHz}$ frequency ranges of RF emission from a plasma produced by laser-matter interactions, respectively [9]. It follows that experiments in previous 
researches mainly aimed at the investigation of plasma radiation characteristics through near-field measurement, where the experiment condition was rather ideal. However, the measuring condition of far-field discharge plasma target DOA estimation in an open space is more complicated, and the subsequent signal processing progress calls for much information of received signals. Therefore, it is of great importance to apply a receiving equipment designed specifically based on the radiation characteristic of spark discharge plasma.

Given the above, an experiment of spark discharge plasma DOA estimation was operated and the results were discussed. The paper is organized as follows: In Section 2, the experimental setup for DOA estimation of spark discharge plasma is introduced, and a combined time-domain antenna for signal receiving is designed based on previously proposed spark discharge model. Moreover, experimental results are illustrated and compared with simulation results in Section 3. Finally, conclusions are drawn in Section 4.

\section{Experimental Setup}

2.1. Experimental Arrangement. The experiment was carried out in an open space, and Figure 1(a) depicts the experimental setup of the outdoor spark discharge plasma target DOA estimation. The experiment setup consists of two parts, namely, spark discharge source circuit and measuring part.

The spark discharge circuit was the same as the one we used in our previous paper [5], which is a gas switch with an RLC second-order circuit (also known as a resonant circuit), as shown in the upper left frame in Figure 1(a). The highvoltage power supply can output a high-voltage direct current (HVDC) up to $40 \mathrm{kV}$. The gas switch, as shown in Figure 1(b), consists of two sphere electrodes, an insulated shell, and gas nozzle. The insulating gas remained changed at 0.1 PMa air in the entire experiment, with a vacuum pump blowing out the exhausted gas every four single repeat experiments. The circuit was charging by the HVDC, and once the charging voltage reached the threshold, spark discharge took place. RF EMPs were generated from the discharge process as soon as the breakdown of gas switch happened and then radiated to free space. Photograph of one single spark discharge process is shown in Figure 1(c).

As for the measuring part on the ground, four same combined time-domain antennas were set on the corner of a square, functioning as a receiving array. Each antenna element was connected to a channel of a four-channel digital oscilloscope (DPO90604 A) through shielded cable. The photograph of the measuring part is shown in Figure 1(d). It should be pointed out that the antenna elements can be placed randomly as far as the baselines are not all parallel; the specific theoretical reason will be explained in Section 2.2. Distance between the antenna array and discharge source is around $320 \mathrm{~m}$ and slightly varies from different test points. When the antennas received the radiated EMPs from spark discharge source, received signals were sent through each channel and then digitalized at a rate of $10 \mathrm{GHz}$ after the oscilloscope was triggered. They are eventually used in post-signal-processing section to estimate the DOA of discharge target using multiple baseline wideband timedomain interferometry. Each single measurement was repeated for 10 times.

2.2. Antenna Design. Unlike traditional active radar target detection, there is little prior information about received signal in passive DOA estimation. Therefore, the estimation performance is strongly related to the receiving quality of signals. In the specific situation discussed in this paper, the radiated EMPs from spark discharge plasma tend to be ultrawideband signals, which makes an antenna with ultrawideband frequency range more suitable as receiving equipment, and can obtain more signal information. However, according to previous research [5], the lower cutoff frequency of radiated EMPs from spark discharge is just a few $\mathrm{MHz}$, and to satisfy the transmission needs in such a low-frequency band, the size of an antenna will be too large to be applied in practice. Therefore, in order to achieve the detection purpose in actual world, a combined antenna is specifically designed based on the radiation characteristics of EMPs from the target.

In our previous study [5], the power of radiated EMPs from spark discharge plasma is mainly between 0 and $200 \mathrm{MHz}$, and a few are over $200 \mathrm{MHz}$. The spark discharge process was separated into two main processes, namely, transient discharge process and subsequent energy releasing process. A combined dipole-loop antenna model was proposed to simulate the whole radiation process of EMPs from spark discharge plasma, where the dipole antenna and loop antenna are used to depict the radiation process of EMP during the discharge transient process and subsequently energy releasing process, respectively. Therefore, based on the proposed model of spark discharge process, a combined time-domain antenna with the ideal operating frequency band and also a convenient size is designed, as shown in Figure 2. The combined antenna consists of three different structures, corresponding to the dipole antenna and the loop antenna in mentioned spark discharge model, respectively. The first structure in red frame is designed with the reference to a TEM horn antenna, which is a wildly used time-domain antenna $[10,11]$ The characteristic impedance of a horn antenna is gradual, so the reflection inside the antenna is very small. Therefore, the radiated EMPs in open space can be coupled and propagated through the antenna in transverse electromagnetic (TEM) mode, which is the propagation mode with the least waveform distortion for timedomain signals. However, the reflection inside the antenna cannot be eliminated completely, and, in order to minimize the influence of reflections, the planar conductors were designed to be exponentially tapered $[12,13]$. When the combined antenna works, the TEM horn antenna part can be regarded as an open transmission line, which is equivalent to a dipole antenna. The second structure in blue frame is a low-frequency compensation circuit; it consists of three conductors that connect the horn from the side rear. This part of the combined antenna improves the low-frequency transmission and reception characteristics of the TEM horn antenna by connecting it to a closed loop and functions as a 
(a)

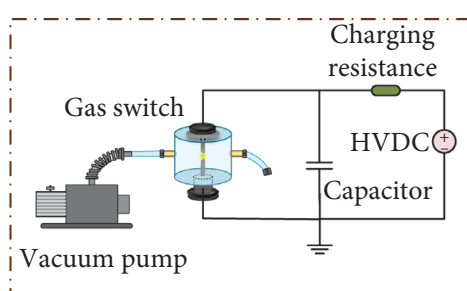

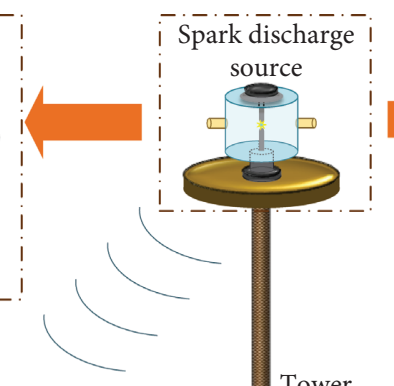

Radiated EMPs

Combined

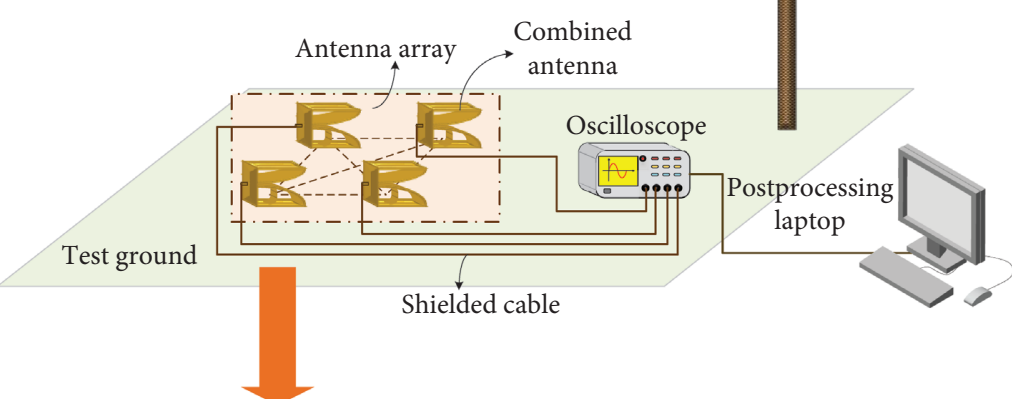

Tower

Tow

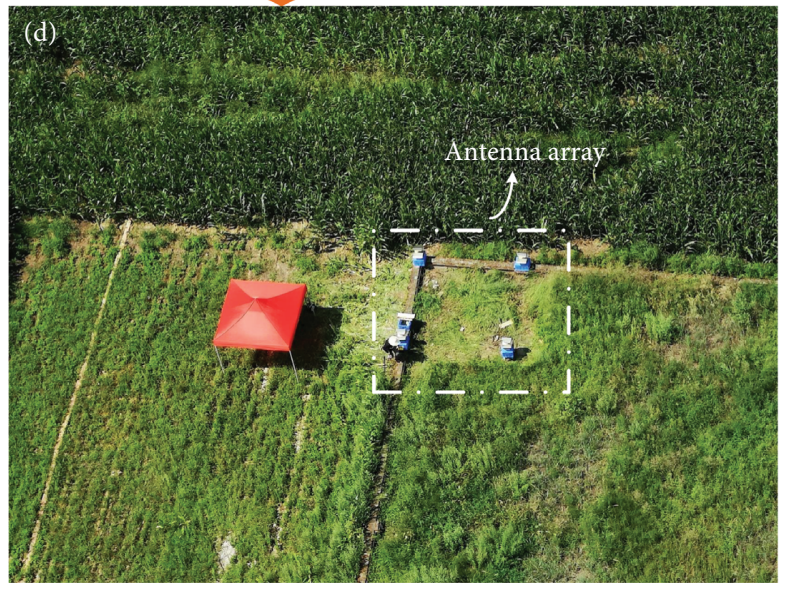

FIGURE 1: Experimental setup.

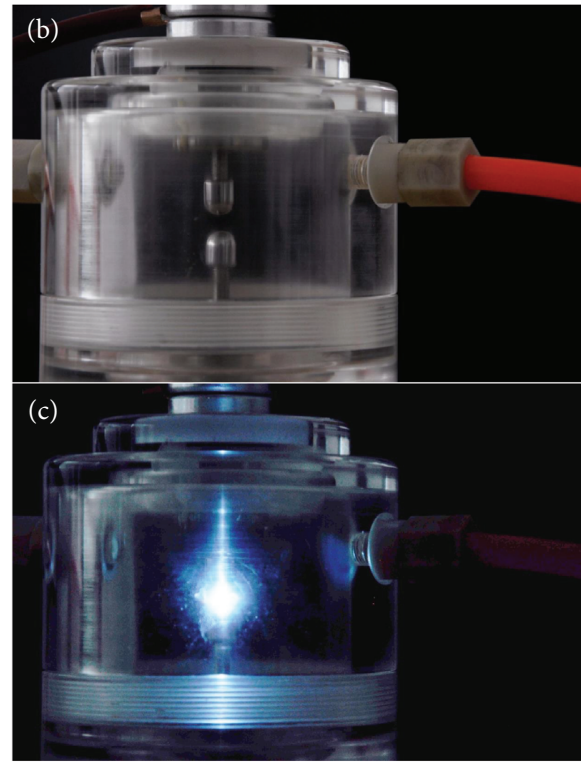

Low-frequency

compensation circuit

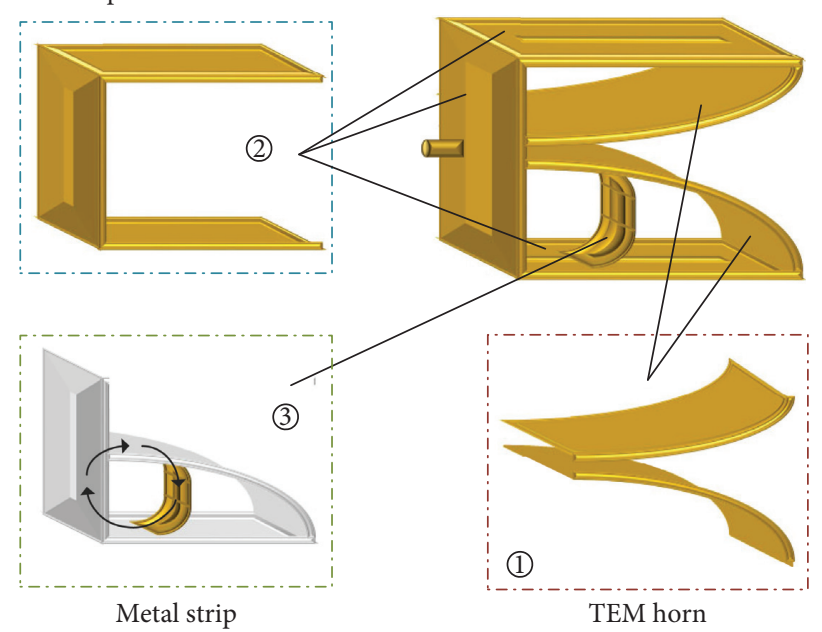

FIgURE 2: Structure of combined time-domain antenna. 
loop antenna. Additionally, a metal strip is added as the third structure to form another loop circuit, as shown in the green frame, and functions as an additional loop antenna. By combining the three structures above, the combined antenna can meet the design requirement in low-frequency band within an acceptable size.

The reception feature of a time-domain antenna is generally described by the impulse response $h(t)$. [14, 15] The designed time-domain antenna shows good performance with the height and width of $46 \mathrm{~cm}$. In time domain, it has an impulse response $h(t)$ with full width at half maximum (FWHM) of $284 \mathrm{ps}$, as shown in Figure 2, and the mid-band effective height is $0.13 \mathrm{~m}$, which is obtained from

$$
h_{\mathrm{eff}}=\sqrt{\frac{50 \Omega}{377 \Omega}} h_{a}=\frac{h_{a}}{2.75},
$$

where $h_{a}=\int h(t) \mathrm{d} t$ is the impulse area. Moreover, the transfer function of combined time-domain antenna in frequency domain is presented in Figure 3(b); it can be seen that the $3 \mathrm{~dB}$ frequency band spans from $0 \mathrm{MHz}$ to $1500 \mathrm{MHz}$, which is an ultra-wideband frequency band, and the low-end cut-off frequency meets the design requirement of receiving the radiated EMPs from spark discharge plasma.

The 3D antenna pattern at different frequency is shown in Figure 4. In frequency band of $100 \sim 200 \mathrm{MHz}$, the combined time-domain antenna is able to radiate and receive the signals from every incident angle, with a maximum radiation direction at $\left(0^{\circ}, 0^{\circ}\right)$. Meanwhile, in higher frequency band, TEM horn antenna starts to dominate the radiation pattern; it can be seen from Figure $4(\mathrm{~d})$ that the radiation gain is more concentrated on the main beam.

To a certain extent, voltage standing wave ratio (VSWR) can also reflect the reception feature of a time-domain receiving antenna. As shown in Figure 5, the combined antenna satisfies VSWR $<2.5$ in the frequency band of $150 \sim 1000 \mathrm{MHz}$, which means that, in this frequency band, the antenna is impedance matched the best to the transmission line.
2.3. DOA Estimation Method. Wideband interferometry is a commonly accepted method in passive detection, such as lightning locating [16-18]. Therefore, it is decided to be applied in signal processing section of the DOA system. A brief introduction of multiple baseline wideband time-domain interferometry will be demonstrated below.

As shown in Figure 6, an antenna array is formed by several antenna elements and is located on the xoy-plane; the coordinates of the elements are $\left(x_{1}, y_{1}, z_{1}\right),\left(x_{2}, y_{2}, z_{2}\right), \ldots,\left(x_{i}\right.$, $\left.y_{i}, z_{i}\right), \ldots,\left(x_{n}, y_{n}, z_{n}\right)$, respectively. Additionally, in order to achieve two-dimensional DOA estimation, the antennas should be noncollinear. With $n$ elements, $C_{n}^{2}$ baselines can be formed between each pair of antennas, and the baseline vector $\mathbf{d}_{m n}$ between the $m$-th and the $n$-th antenna element can be expressed as follows:

$$
\mathbf{d}_{m n}=\left(x_{n}-x_{m}, y_{n}-y_{m}, z_{n}-z_{m}\right)
$$

The basic theory of interferometry is to estimate the DOA of radiation target with the phase difference between the arriving signals at each antenna element. The phase difference between two antennas in the array can be expressed as

$$
\Delta \varphi_{m n}=\frac{2 \pi f}{c} \mathbf{d}_{m n} \mathbf{r}=\frac{2 \pi f}{c}\left[\left(x_{n}-x_{m}\right) u+\left(y_{n}-y_{m}\right) v\right]=k_{m n} f
$$

where $f$ is the frequency of received signal, $r$ stands for the distance between target and antenna array, and $c$ is the light speed; $u=\sin \mathrm{Az} \cos \mathrm{El}, v=\cos \mathrm{Az} \cos \mathrm{El}$ are the direction cosines of discharge target $P$; "Az" and "El" stand for the azimuth angle and elevation angle of the discharge source, respectively, and $k_{m n}=(2 \pi / c)\left[\left(x_{n}-x_{m}\right) u+\left(y_{n}-y_{m}\right) v\right]$ is the slope of $\Delta \varphi_{m n}-f$ linear curve when received signal is wideband, and it can be obtained by curve fitting algorithm. Then a linear equation set about the direction cosines can be written as

$$
\begin{gathered}
{\left[\left(x_{n}-x_{m}\right) u+\left(y_{n}-y_{m}\right) v\right]=\frac{c}{2 \pi} k_{m n} \Longleftrightarrow\left[\begin{array}{cc}
x_{2}-x_{1} & y_{2}-y_{1} \\
x_{3}-x_{1} & y_{3}-y_{1} \\
\vdots & \vdots \\
x_{3}-x_{2} & y_{3}-y_{2} \\
x_{4}-x_{2} & y_{4}-y_{2} \\
\vdots & \vdots \\
v
\end{array}\right]=\frac{c}{2 \pi}\left[\begin{array}{c}
u \\
k_{13} \\
\vdots \\
k_{23} \\
k_{24} \\
\vdots \\
x_{n}-x_{n-1} \\
y_{n}-y_{n-1}
\end{array}\right],} \\
\Longleftrightarrow \mathbf{A}\left[\begin{array}{c}
u \\
v
\end{array}\right]=\frac{c}{2 \pi} \mathbf{B},
\end{gathered}
$$




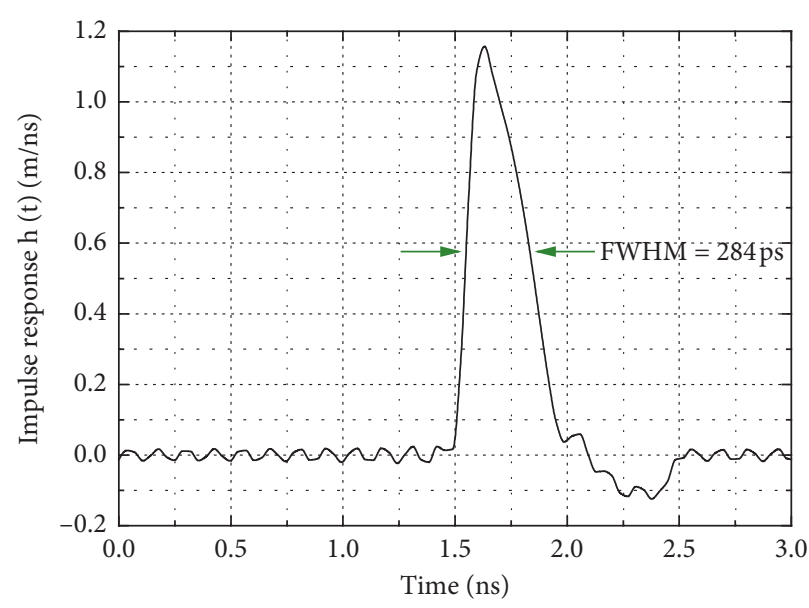

(a)

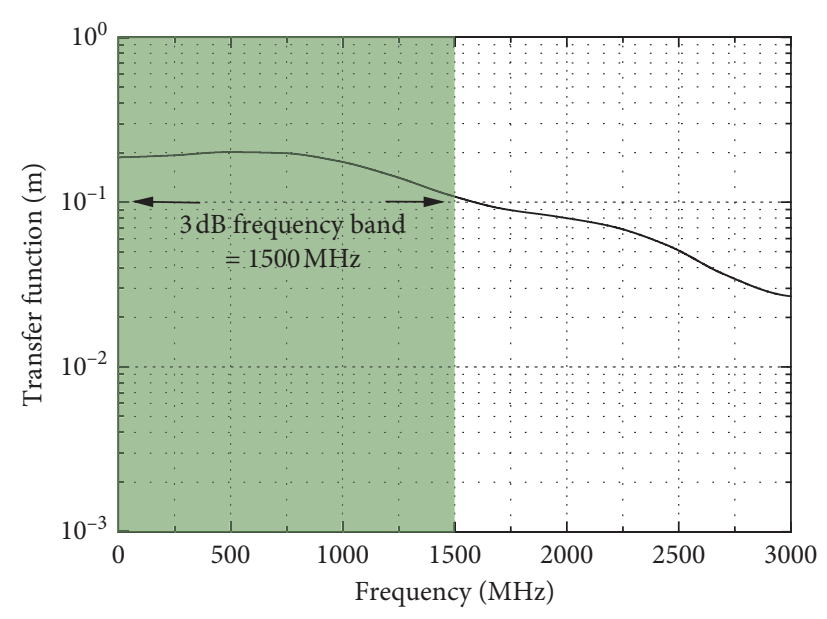

(b)

FIgURE 3: The performance of combined time-domain antenna. (a) Impulse response in time domain. (b) Transfer function in frequency domain.

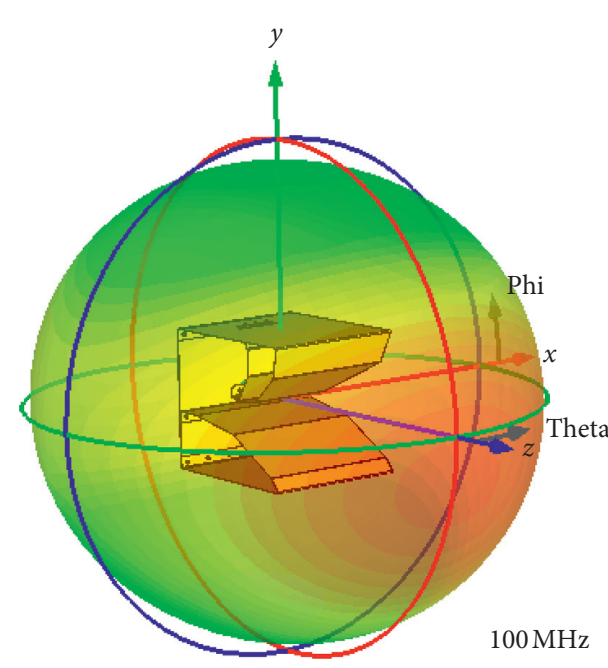

(a)

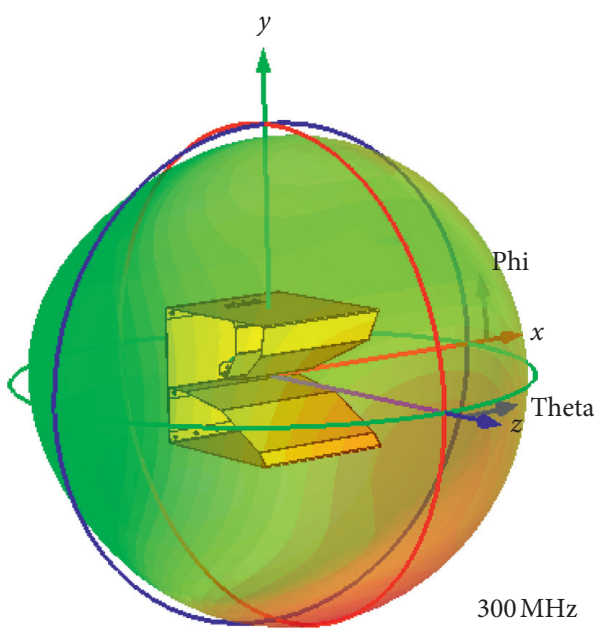

(c)
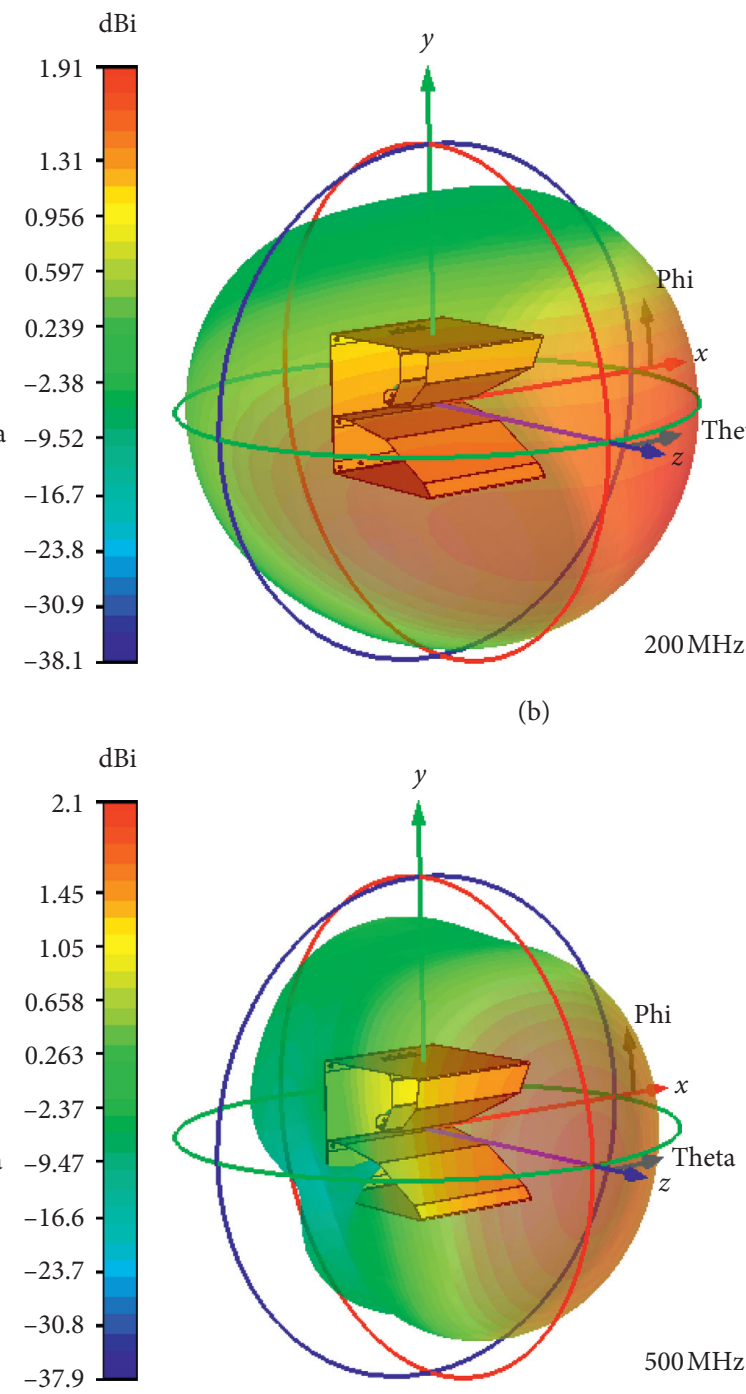

(d)

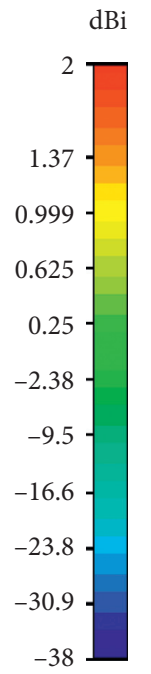

(b)

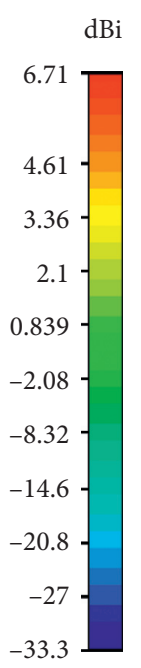

Figure 4: 3D antenna pattern at different frequency. 


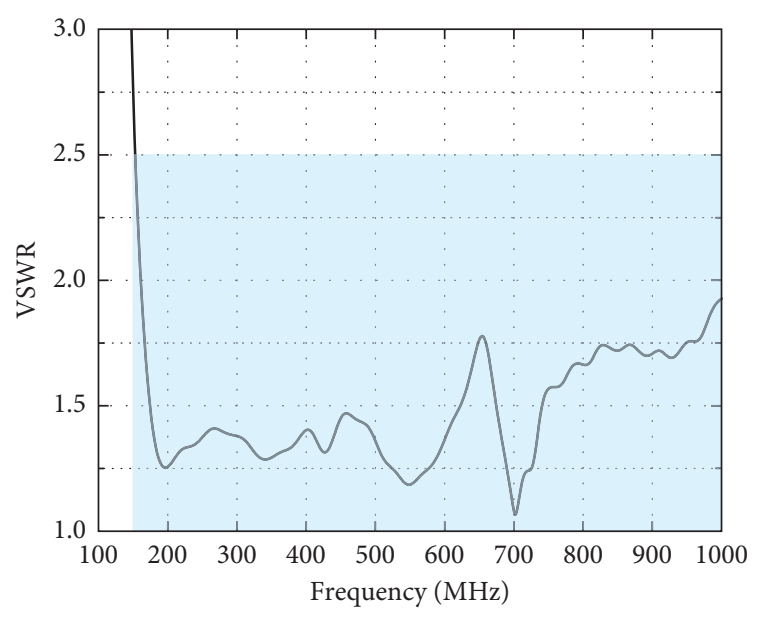

Figure 5: VSWR of combined time-domain antenna.

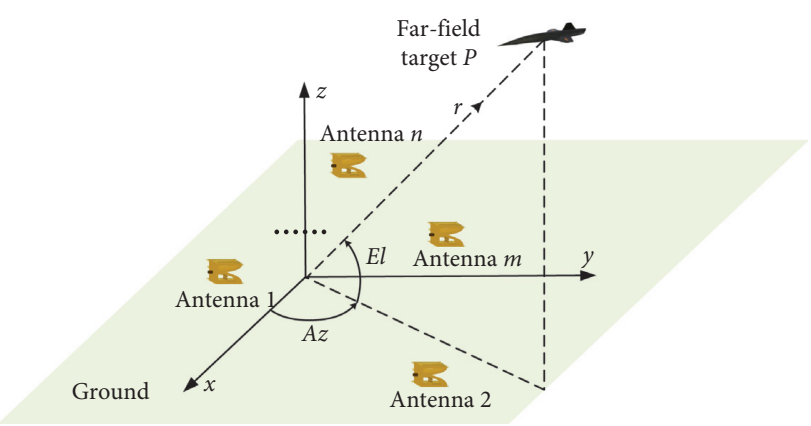

Figure 6: Multiple baseline interferometry.

where $\mathbf{A}$ stands for the antenna position parameters and $\mathbf{B}$ stands the slopes. When more than two baselines are used, the direction cosines $u$ and $v$ can be solved with least square solution:

$$
\left[\begin{array}{l}
u \\
v
\end{array}\right]=\frac{c}{2 \pi}\left(\mathbf{A}^{T} \mathbf{A}\right)^{-1} \mathbf{A}^{T} \mathbf{B},
$$

and, finally, the elevation and azimuth angles can be obtained by

$$
\left\{\begin{array}{l}
\mathrm{El}=\cos ^{-1}\left(\sqrt{u^{2}+v^{2}}\right), \\
\mathrm{Az}=\tan ^{-1}\left(\frac{v}{u}\right)
\end{array}\right.
$$

According to above analysis, the incident angles can be estimated only if we figure out the slope $k_{m n}$ of $\Delta \varphi_{m n}-f$ curve. Meanwhile, in wideband interferometry, the phase undergoing beyond $\pm \pi$ will be wrapped since the output angle of a phase discriminator is limited within $[-\pi, \pi]$, so the exact value of $k_{m n}$ is hard to obtain. To solve this ambiguity problem, certain unwrapping algorithm was come up using the continuous frequency components of a wideband signal [19]. However, the process of phase unwrapping and curve fitting in frequency domain can cause certain system error, and, in order to avoid this problem, equivalent operation will be accomplished in time domain directly. When signal arrives at antenna array, the arrival time at each antenna element differs from others, as a result of the path difference; then, $k_{m n}$ can be calculated in time domain as

$$
k_{m n}=2 \pi \Delta t_{m n}
$$

where $\Delta t_{m n}$ is the time delay between the arrival times of signals at antennas $m$ and $n$. So matrix B in equation (4) can be written as

$$
\mathbf{B}=2 \pi\left[\begin{array}{lllllll}
\Delta t_{21} & \Delta t_{31} & \cdots & \Delta t_{32} & \Delta t_{42} & \cdots & \Delta t_{n(n-1)}
\end{array}\right]^{T} .
$$

Therefore, instead of calculating the slope of $\Delta \varphi_{m n}-f$ curve in frequency domain, the DOA estimation can be completed in time domain with the time delay $\Delta t_{m n}$, which can be obtained by cross-correlating the two received signals. Suppose that the received signals of antennas $m$ and $n$ are denoted as $s_{m}(t)$ and $s_{n}(t)$, respectively. Then the crosscorrelation is defined as

$$
r_{m n}(\tau)=E\left[s_{m}(t) s_{n}(t+\tau)\right]
$$

where $E[*]$ stands for the mathematical expectation. The peak of $r_{m n}(\tau)$ appears when $\tau=\Delta t_{m n}$; therefore, the time delay can be estimated by searching the peak position.

\section{Experimental Results and Discussion}

The experiment measured the radiated EMPs from a single spark discharge process. When spark discharge happens, discharge pulses started to be radiated to open space. Corresponding research of spark discharge has been accomplished. The results show that the EMPs radiated from a spark discharge plasma are ultra-wideband signals with a frequency band of $0 \sim 200 \mathrm{MHz}$, and the energy in $100 \sim 200 \mathrm{MHz}$ mainly comes from the transient discharge process, while the energy in $0 \sim 100 \mathrm{MHz}$ comes from the subsequent energy releasing process. A time-domain waveform of radiation signal in our combined receiving antenna is shown in Figure 7(a); typical ultra-wideband pulse feature can be observed from the received waveform. Fast Fourier transform (FFT) was then applied on the received signal, and the amplitude-frequency curve is illustrated in Figure 7(b). The energy of received signal mainly concentrates on the frequency band of $0 \sim 200 \mathrm{MHz}$, which agrees well with the radiation band of EMPs from the discharge source. The above results confirm the performance of combined time-domain antenna receiving radiated EMPs from spark discharge plasma.

In order to study the DOA estimation performance at different conditions, the receiving antenna array was placed at different locations, and four aperture sizes were applied at each location point. The estimation performance is examined in terms of the root mean square error (RMSE), which is defined as 


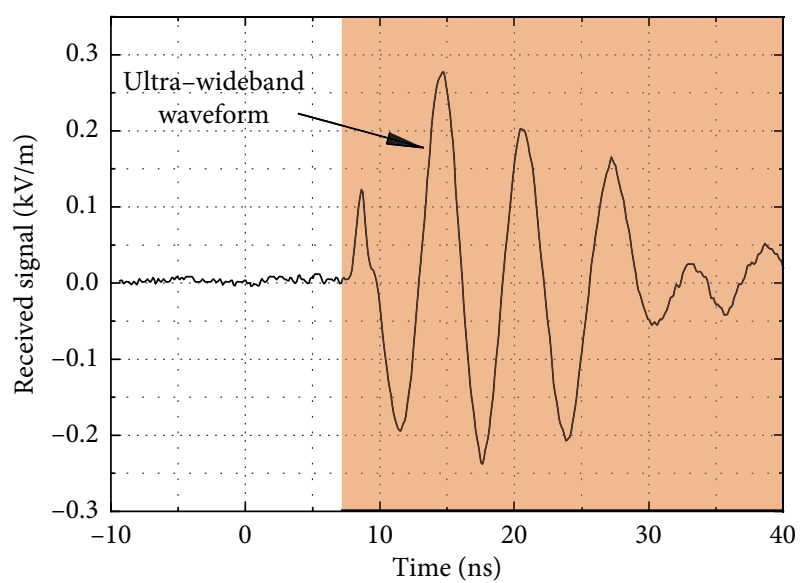

(a)

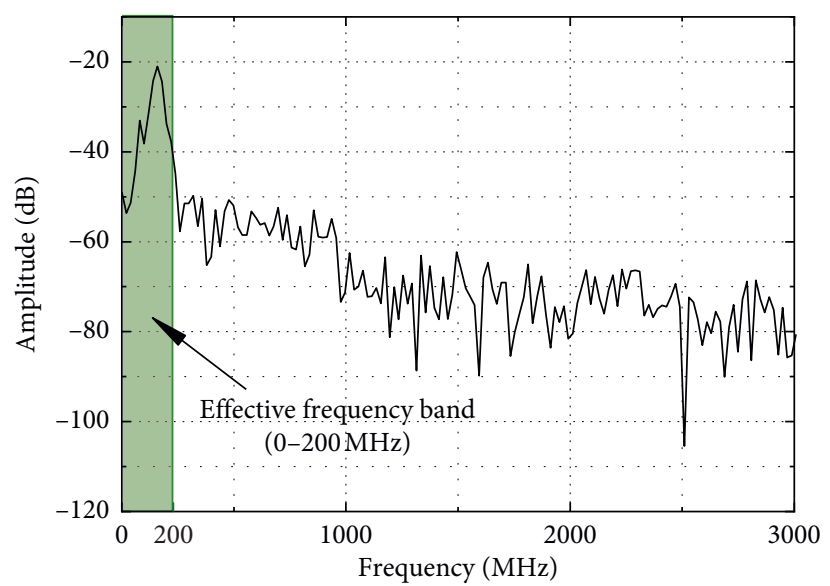

(b)

Figure 7: Received signal in (a) time domain and (b) frequency domain.

$$
\text { RMSE }=\sqrt{\frac{1}{N} \sum_{i=1}^{N}\left(\widehat{\theta}_{i}-\theta\right)^{2}},
$$

where $\theta$ is the true DOA value of incident signal, and, in 2dimensional DOA estimation, it can represent both the elevation angle and azimuth angle. $\widehat{\theta}$ is the ith estimation result of DOA, and $N$ is the number of repeated experiments. The DOA estimation method is the multiple baseline wideband time-domain interferometry introduced in Section 2.3, and the algorithm flow is shown in Figure 8.

Firstly, the influences of elevation and azimuth angles of radiated EMP signal from spark discharge plasma source were investigated. Figure 9(a) shows the RMSE of experimental estimation results under different elevation angles when the side length of antenna aperture is $4 \mathrm{~m}$ and azimuth angle is 168.88 degrees. It can be seen that when elevation angle increases from 24.66 degrees to 32.82 degrees, the estimation RMSE of elevation angle declines from 2.07 degrees to 0.79 degrees, while the estimation RMSE of azimuth angle increases slightly from 0.84 degrees to 1.46 degrees. Simulation under different elevation angles is also carried out with the same DOA estimation method. During the simulation, a typical measured signal in the experiment was used as a source signal, and 1000 trails of Monte Carlo simulation were operated under each angle (24.66 degrees to 32.82 degrees) when side length of antenna aperture is $4 \mathrm{~m}$ and azimuth angle is 168.88 degrees. The result is also presented in Figure 9(a), showing that the experimental results share the same variation trend with the simulation results. Reasons for this effect of elevation angle are explained as follows: equation (3) describes the principle of multiple baseline wideband interferometry; when the positions of all the antennas and other variables are fixed, the phase difference is a function of the incident angles "El" and "Az," as shown in the following equation:

$$
\Delta \varphi=f(\mathrm{El}, \mathrm{Az}) .
$$

Therefore, the relationship between RMSE of elevation angle $\sigma_{\mathrm{El}}$ and the RMSE of phase difference $\sigma_{\Delta \varphi}$ can be expressed as

$$
\sigma_{\Delta \varphi}=\left|\frac{\partial \Delta \varphi}{\partial \mathrm{El}}\right| \cdot \sigma_{\mathrm{El}}
$$

Equation (12) can be rewritten as

$$
\sigma_{\mathrm{El}}=\left|\frac{\partial \Delta \varphi}{\partial \mathrm{El}}\right|^{-1} \cdot \sigma_{\Delta \varphi}
$$

According to equation (3), $|(\partial \Delta \varphi / \partial \mathrm{El})|^{-1} \propto(\sin \mathrm{El})^{-1}$. Similarly,

$$
\sigma_{\mathrm{Az}}=\left|\frac{\partial \Delta \varphi}{\partial \mathrm{Az}}\right| \cdot \sigma_{\Delta \varphi} \propto(\cos \mathrm{El})^{-1} \sigma_{\Delta \varphi}
$$

where $\sigma_{\Delta \varphi}$ is related to time delay estimation and is only determined by system error. So, according to equation (13), when elevation angle increases from 0 degrees to 90 degrees and azimuth angle remains still, the estimation RMSE of elevation angle decreases, while that of azimuth angle becomes higher according to equation (14). For clearer observation, we expand the simulation angles to 0 90 degrees, and the result can be found in Figure 9(b). The simulation result agrees with the change trend of experimental result when elevation angle increases, and, in particular, a relatively large RMSE in azimuth angle estimation can be observed when discharge source is very close to the top of the antenna array. However, in practical applications, the DOA of a target nearly direct above can be determined only by the estimation result of its elevation angle, which is fairly precise under this situation.

Furthermore, experiments under different azimuth angles were also carried out, and the result is illustrated in Figure 10(a). Unlike the results under different elevation angles, the RMSE of two incident angles presents an irregular change within 2 degrees when azimuth angle increases from 155 degrees to 179 degrees. Simulations were operated with 1000 Monte Carlo trails under each azimuth angle (155 degrees to 179 degrees) when side length of antenna aperture is $4 \mathrm{~m}$ and elevation angle is 30.31 degrees; as presented in the zoom-in frame in Figure 10(a), the RMSE results also oscillate randomly as the experimental results 


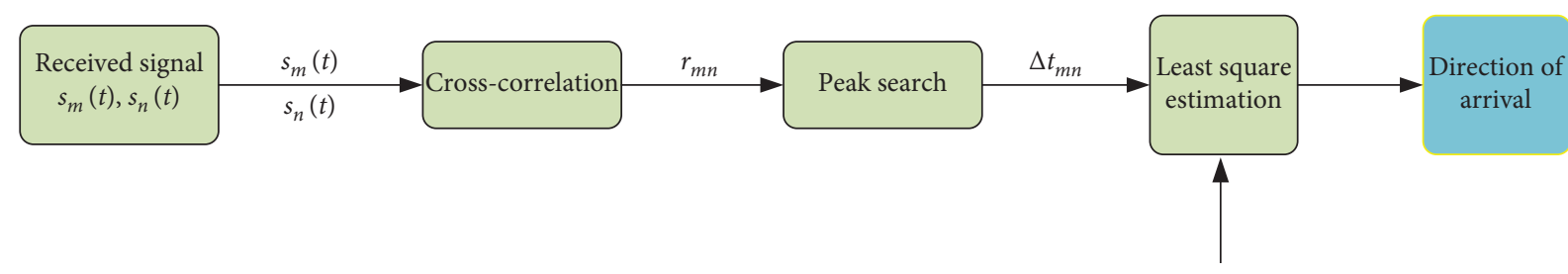

Antenna position parameters

$\left(x_{1}, y_{1}, z_{1}\right),\left(x_{2}, y_{2}, z_{2}\right), \cdots,\left(x_{\mathrm{i}}, y_{\mathrm{i}}, z_{\mathrm{i}}\right), \cdots,\left(x_{\mathrm{n}}, y_{\mathrm{n}}, z_{\mathrm{n}}\right)$

FIGURE 8: Flow diagram of multiple baseline wideband time-domain interferometry.

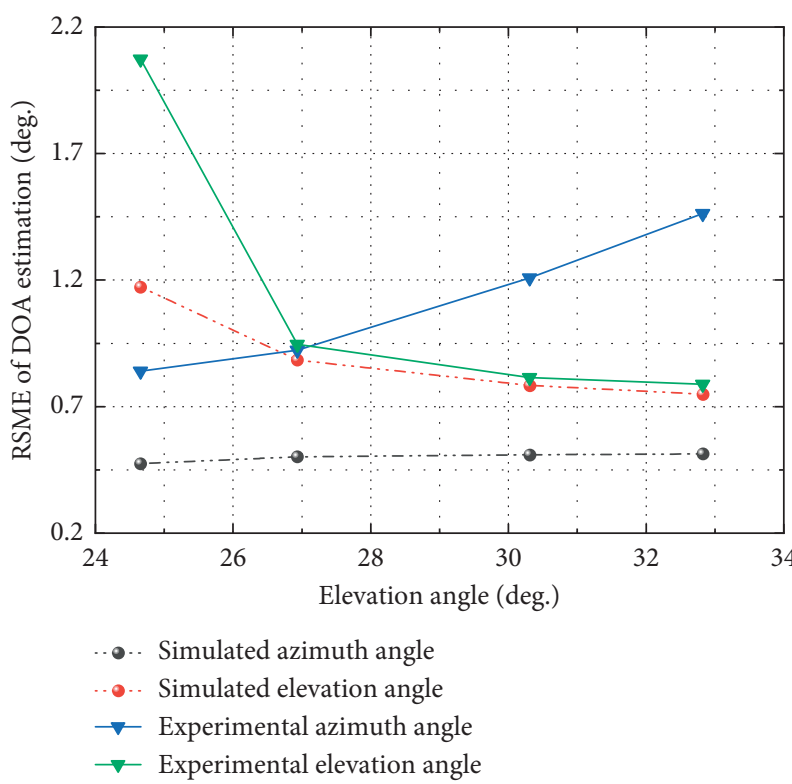

(a)

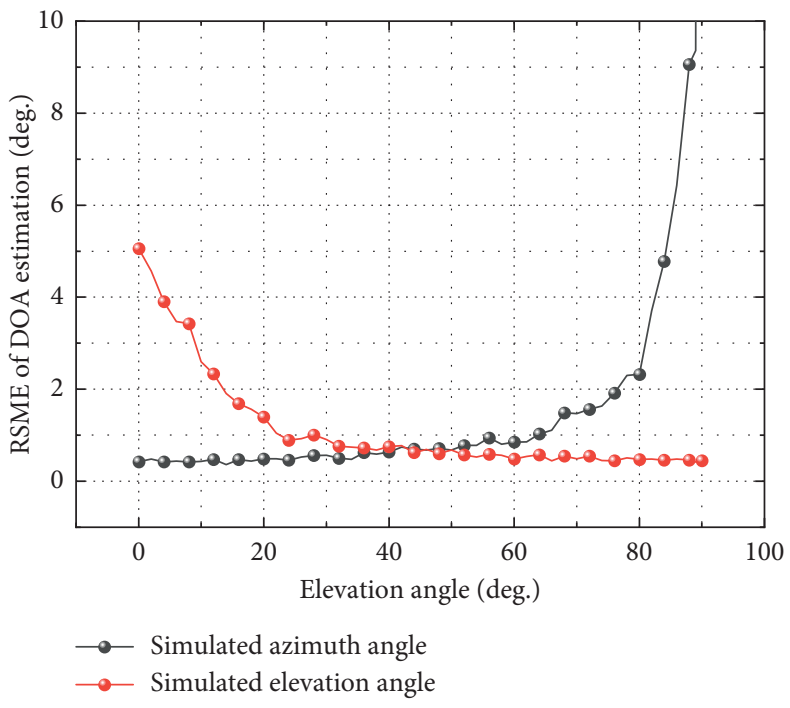

(b)

FIGURE 9: RMSE of DOA estimation under different elevation angles when side length of antenna aperture is $4 \mathrm{~m}$ and azimuth angle is 168.88 degrees. (a) Comparison between the experimental results and simulation results. (b) Simulation results under elevation angle of 0-90 degrees.

but only in a smaller range. Also, the simulation results under azimuth angle of -180 degrees to 180 degrees are shown in Figure 10(b), and small particular effect of azimuth angle on DOA estimation can be found; therefore, no specific treatment is needed in practical measuring when the azimuth angle is changed.

Moreover, experiments under different aperture sizes were operated. Figure 11 presents the estimation RMSE result under the aperture side lengths of $1 \mathrm{~m}, 2 \mathrm{~m}, 3 \mathrm{~m}$, and $4 \mathrm{~m}$. When the side length of the rectangular aperture antenna array is $1 \mathrm{~m}$, the estimation RMSE of two incident angles are up to 5.27 degrees and 5.77 degrees. With the increase of aperture size, the estimation performance is improved effectively, and the RMSE of elevation angle and azimuth angle are reduced to 0.95 and 0.92 degrees, respectively. Simulation results under different apertures when elevation angle is 26.93 degrees and azimuth angle is 168.88 degrees can be found in Figure 11 as well; the RMSE of DOA estimation gradually declines with the increase of aperture size. Reasons can be explained by the basic principle equation of interferometry:

$$
\Delta \varphi=\frac{2 \pi}{\lambda} \Delta l=\frac{2 \pi f}{c} d \cos \theta,
$$

where $\Delta l$ is the path difference of two arriving signals, $d$ is the baseline length, $\lambda$ is the wavelength of received signal, $f$ is the signal frequency, and $\theta$ is the incident angle, which, in this case, can represent both the elevation angle and azimuth angle. After derivation calculus on incident angle $\theta$ to both sides of equation (15), the following equation can be obtained:

$$
\frac{d \Delta \varphi}{d \theta}=-\frac{2 \pi f}{c} d \sin \theta
$$

Then, the estimation error of $\theta$ can be expressed as

$$
\sigma_{\theta}=\left|\frac{\mathrm{d} \Delta \varphi}{\mathrm{d} \theta}\right|^{-1} \cdot \sigma_{\Delta \varphi}=\left[\frac{c}{2 \pi f \sin \theta} d^{-1}\right] \sigma_{\Delta \varphi}
$$




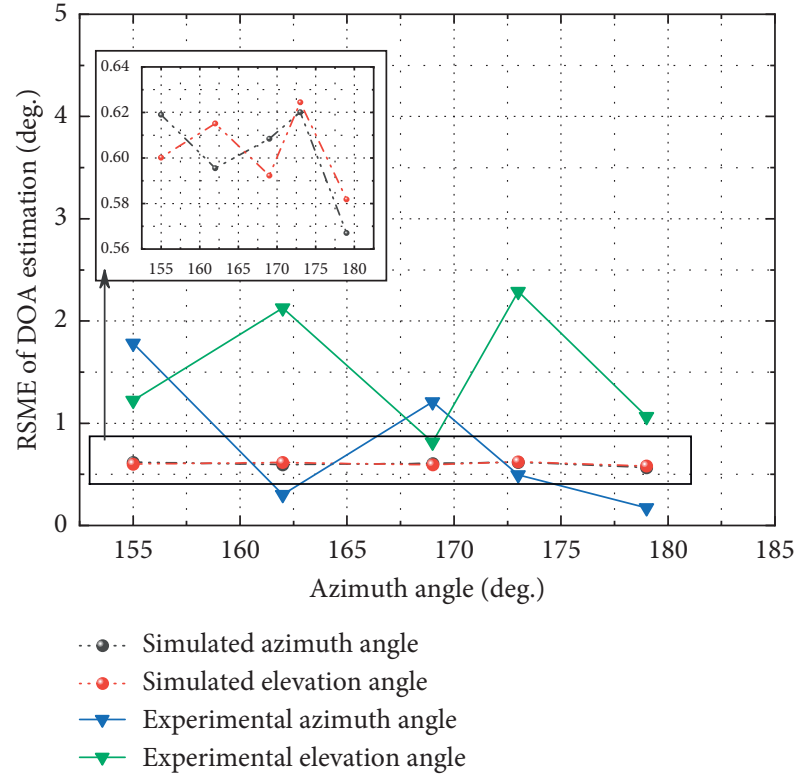

(a)

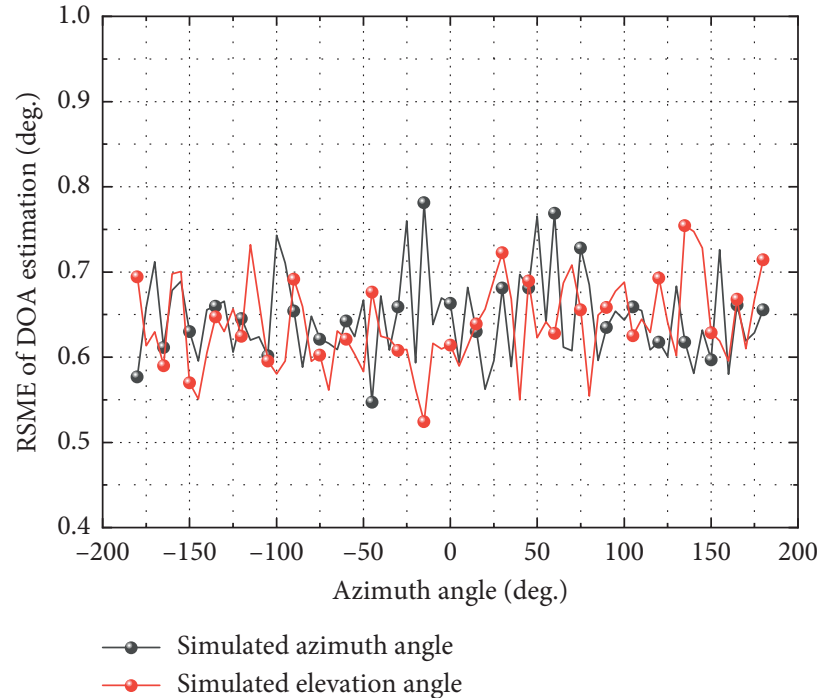

(b)

FIGURE 10: RMSE of DOA estimation under different azimuth angles when side length of antenna aperture is $4 \mathrm{~m}$ and elevation angle is 30.31 degrees. (a) Comparison between the experimental results and simulation results. (b) Simulation results under azimuth angle of $-180-180$ degrees.

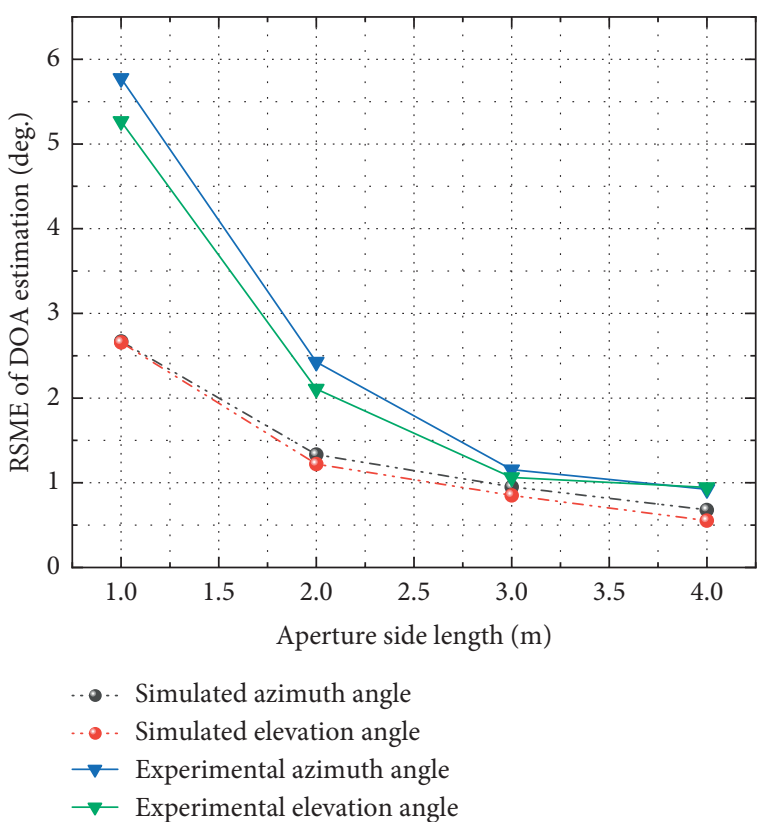

FIGURE 11: RMSE of DOA estimation under different aperture sizes when elevation angle is 26.93 degrees and azimuth angle is 168.88 degrees.

where $\sigma_{\theta}$ and $\sigma_{\Delta \varphi}$ are the RMSE of incident angle estimation and phase difference. As is shown in equation (17), the estimation error of incident angle is inversely related to baseline length $d$. Therefore, to achieve more accurate discharge source DOA estimation, it is worth to consider increasing the aperture size within an allowable range. Specifically, the RMSE of experimental results under the aperture side length of $1 \mathrm{~m}$ is much larger than that of the simulation results. One main reason can be assumed to be the mutual coupling effect between two antenna elements. When the distance between two antennas is $1 \mathrm{~m}$, the mutual coupling effect can become stronger and brings about more distortion in received signal. In particular, the radiated EMPs from spark discharge oscillate with a high frequency, so the distortion in time-domain waveform can cause a larger variance in time delay estimation. Mutual coupling effect becomes weaker under larger baseline length; therefore, in practical measurement, small antenna elements distance should be avoided.

Finally, the comparison with Cramér-Rao Lower Bound (CRLB) is shown in Figure 12. The DOA estimation variance of the interferometer is governed by the uncertainty of the time delay estimation. Therefore, the theoretical lower bound of the angle estimation uncertainty with signal-tonoise ratio (SNR) for both azimuth angle and elevation angle is $[18,20,21]$

$$
\sigma^{2}=\frac{c}{d} \frac{3}{8 \pi^{2}} \frac{1+2 \mathrm{SNR}}{\mathrm{SNR}^{2}} \frac{1}{\operatorname{BT}\left(3 f_{c}^{2}+B^{2} / 4\right)} .
$$

It can be seen from Figure 12 that the DOA estimation performance of multiple wideband interferometry is better when SNR > $-5 \mathrm{~dB}$.

However, the RMSE of experimental results is larger than that of the simulation results; several possible causes can be assumed:

(a) Coupling effect among antenna elements: When antennas are close to each other, mutual coupling effect can happen and cause certain distortion in the time-domain waveform of received signals. The 


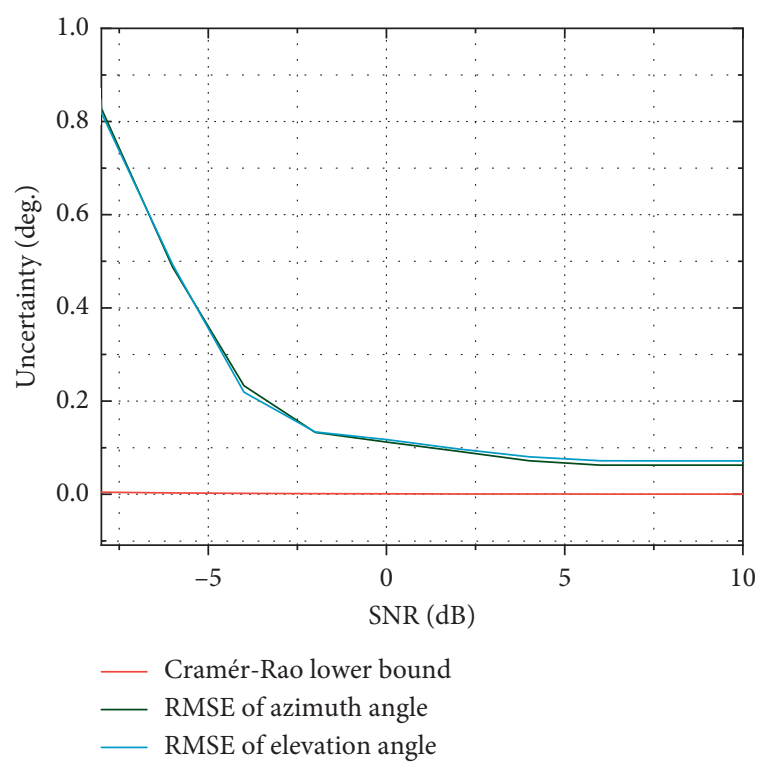

Figure 12: Comparison of DOA estimation RMSE and CRLB.

distortion can bring about error during the time delay estimation and eventually lead to higher RMSE in incident angle estimation.

(b) Measuring error in antenna position: As is introduced in Section 2.3, besides the information of received signals, the DOA estimation also relies on the position information of each antenna element. So, the measuring error in antenna position can also make the practical RMSE higher than simulation.

(c) Imbalance among antenna elements: Although we have tried to make every combined time-domain antenna the same, inevitable imbalance still exists among those antenna elements due to various reasons, which can influence the time delay estimation and degrade the DOA estimation performance.

\section{Conclusions}

To validate the idea of utilizing the radiated EMPs from spark discharge plasma in passive detection, an experiment on DOA estimation for atmospheric spark discharge plasma is carried out. To receive the radiated EMPs from discharge process effectively, a combined time-domain antenna is designed based on the model of spark discharge process. The main structure of combined antenna refers to the TEM horn antenna, and, in order to improve the receiving performance in low-frequency band, a low-frequency compensation circuit and an additional metal strip are added to the antenna structure. When the combined antenna is working under the expected frequency band, the TEM horn part can be equivalent to a dipole antenna, and the low-frequency compensation circuit and the metal strip can be regarded as two loop antennas, as proposed in the spark discharge model. Wideband time-domain interferometry is applied in the signal processing part to estimate the DOA of spark discharge target. Finally, the experimental results under different conditions are illustrated and analyzed. Results confirm that the direction information of a spark discharge plasma can be obtained effectively using the DOA estimation system with the combined time-domain antenna. It can also be observed that when the elevation of discharge target increases, the estimation performance of elevation angle becomes better, while the estimation of azimuth angle gets worse. However, the azimuth angle of the target has small influence on the DOA estimation results. Additionally, by larger aperture size of antenna array, the estimation performance can be improved.

\section{Data Availability}

The CSV data used to support the findings of this study are available from the corresponding author upon request.

\section{Conflicts of Interest}

The authors declare that there are no conflicts of interest regarding the publication of this paper.

\section{Acknowledgments}

This work was supported by China Postdoctoral Science Foundation (no. 2018M633598).

\section{References}

[1] A. J. Illingworth and S. J. Marsh, "Static charging of aircraft by collisions with ice crystals," Revue de Physique Appliquée, vol. 21, no. 12, pp. 803-808, 1986.

[2] J. Rybak and R. J. Churchill, "Progress in reentry communications," IEEE Transactions on Aerospace and Electronic Systems, vol. AES-7, no. 5, pp. 879-894, 1971.

[3] R. Hartunian, G. Stewart, S. Furgason, T. Curtiss, and R. Seibold, "Cause and mitigation of radio frequency (RF) blackout during reentry of reusable launch vehicle," Aerospace Report No. ATR-2007 (5309)-1, The Aerospace Corporation, EI Segundo, CA, USA, 2007.

[4] A. Ohzu and K. Ito, "Characteristics of hard x-ray emission from laser-induced vacuum spark discharges," Journal of Applied Physics, vol. 93, no. 12, pp. 9477-9482, 2003.

[5] J. Yan, L. Xu, W. Tie, D. Jiang, and B. Yan, "Experimental investigation on radiation characteristics of RF electromagnetic pulse from atmospheric spark discharge plasma," Physics of Plasmas, vol. 26, no. 4, Article ID 043301, 2019.

[6] J. S. Pearlman and G. H. Dahlbacka, "Emission of rf radiation from laser-produced plasmas," Journal of Applied Physics, vol. 49, no. 1, pp. 457-459, 1978.

[7] X. Wang, Y. Dai, X. Song, X. Gao, and J. Lin, "Space distribution of radio frequency radiation from air plasma produced by nanosecond pulsed laser," Optics Communications, vol. 450, pp. 155-159, 2019.

[8] B. Forestier, A. Houard, M. Durand et al., "Radiofrequency conical emission from femtosecond filaments in air," Applied Physics Letters, vol. 96, no. 14, Article ID 141111, 2010.

[9] J. A. Aspiotis, N. Barbieri, R. Bernath, C. G. Brown, and B. Y. Cooper, "Detection and analysis of RF emission generated by laser-matter interactions," in Proceedings of Spie the International Society for Optical Engineering, San Diego, CA, USA, March 2006. 
[10] A. R. Mallahzadeh and F. Karshenas, "Modified TEM horn antenna for broadband applications," Progress in Electromagnetics Research, vol. 90, no. 4, pp. 105-119, 2009.

[11] W. Yun, Y. Chen, and L. Fan, "Simulation for several kinds of TEM horn antenna used by NEMP simulator," in Proceedings of the International Applied Computational Electromagnetics Society Symposium, Suzhou, China, August 2017.

[12] M. Khorshidi and M. Kamyab, "New exponential TEM horn antenna with binomial impedance taper," AEU-International Journal of Electronics and Communications, vol. 64, no. 11, pp. 1073-1077, 2010.

[13] A. A. H. Ameri, G. Kompa, and A. Bangert, "Study about TEM horn size reduction for ultrawideband radar application," in Proceedings of the 2011 German Microwave Conference, pp. 1-4, Darmstadt, Germany, March 2011.

[14] E. G. Farr, A Standard for Characterizing Antenna Performance in the Time Domain, Springer New York, New York, NY, USA, 2014.

[15] E. G. Farr, "A power wave theory of antennas," in Proceedings of the IEEE International Conference on Ultra-Wideband, Sydney, Australia, September 2013.

[16] X. M. Shao, D. N. Holden, and C. T. Rhodes, "Broad band radio interferometry for lightning observations," Geophysical Research Letters, vol. 23, no. 15, pp. 1917-1920, 1996.

[17] Z. Kawasaki, R. Mardiana, and T. Ushio, "Broadband and narrowband RF interferometers for lightning observations," Geophysical Research Letters, vol. 27, no. 19, pp. 3189-3192, 2000.

[18] M. G. Stock, M. Akita, P. R. Krehbiel et al., "Continuous broadband digital interferometry of lightning using a generalized cross-correlation algorithm," Journal of Geophysical Research: Atmospheres, vol. 119, no. 6, pp. 3134-3165, 2014.

[19] R. Mardiana and Z. Kawasaki, "Broadband radio interferometer utilizing a sequential triggering technique for locating fast-moving electromagnetic sources emitted from lightning," IEEE Transactions on Instrumentation and Measurement, vol. 49, no. 2, pp. 376-381, 2000.

[20] G. C. Carter, "Coherence and time delay estimation," Proceedings of the IEEE, vol. 75, no. 2, pp. 236-255, 1987.

[21] M. Stock and P. Krehbiel, "Multiple baseline lightning interferometry-improving the detection of low amplitude VHF sources," in Proceedings of the 2014 International Conference on Lightning Protection (ICLP 2014), Shanghai, China, October 2014. 